\title{
Screening-Studie unter Südtiroler Schülern nach Tuberkuloseexposition
}

\author{
Screening of South Tyrolean School Children after Exposure \\ to a Tuberculosis Case
}

Autoren

Institut
P. Kreidl ${ }^{1}$, P. Pretto ${ }^{2}$, G. Morosetti ${ }^{3}$

Epidemiologische Beobachtungsstelle, Assessorat für Gesundheit und Soziales

Überbetrieblicher Pneumologischer Dienst des Sanitätsbetriebes Bozen

Amt für Hygiene und öffentliche Gesundheit, Assessorat für Gesundheit und Soziales eingereicht 1.1. 2006

akzeptiert 25.5. 2006

Bibliografie

DOI 10.1055/s-2006-944250

Pneumologie 2006; 60;

512-518

(c) Georg Thieme Verlag KG Stuttgart $\cdot$ New York.

ISSN 0934-8387

Korrespondenzadresse

Dr. Peter KreidI

Epidemiologische Beobachtungsstelle · Assessorat

für Gesundheit

Freiheitsstr. 23

39100 Bozen

Italien

peter.kreidl@provinz.bz.it

\section{Zusammenfassung}

Hintergrund Während eines Sprachaufenthaltes in Irland hatten 422 Schüler, im Alter zwischen 15 und 17 Jahren, und 44 Begleitpersonen aus der autonomen Provinz Bozen - Südtirol in Italien mit einem irischen, an einer Lungentuberkulose erkrankten Lehrer wiederholt Kontakt. Die Expositionsdauer betrug insgesamt mindestens 10 Unterrichtsstunden. Der Nachweis von Mycobacterium-tuberculosis-Komplex gelang beim Erkrankten zwar in der Sputumkultur, der Ausstrich aber war negativ.

Methode Sofortige Maßnahmen waren die Identifizierung aller Kontakte, Erhebung der Anamnese mit einem Standardfragebogen, Tuberkulinprobe nach Mantoux mit 5IE PPD und eine Thoraxröntgenuntersuchung von Personen mit einer Induration $\geq 5 \mathrm{~mm}$. Im Fragebogen wurden Informationen über demografische Faktoren, Symptome, Impfstatus, Grunderkrankungen, regelmäßige Arzneimitteleinnahme und während der letzten 3 Monate durchgemachte Infektionskrankheiten erhoben. Des Weiteren wurde eine Kontrollgruppe in denselben Schulen mit ähnlichem BCG-Impfstatus und ähnlicher Altersund Geschlechtsverteilung ausgesucht, um den Effekt der Exposition und die Auswirkungen einer zurückliegenden BCG-Impfung und der Umweltmykobakterien abschätzen zu können.

Ergebnisse Die Exposition in Irland war nicht mit einer erhöhten Prävalenz einer Induration $\geq 5 \mathrm{~mm}$ in der multivariaten Analyse assoziiert, jedoch intra-familiärer Kontakt (OR $=3,3 ; 95 \%$ Konfidenzintervall: $1,35-8,07$ ) oder von der Schule A zu kommen (OR $=2,3$; $95 \%$ Konfidenzintervall: $1,19-4,32)$.

Schlussfolgerungen Umfeldkontrollen eines infektiösen Tuberkulosefalls sind wichtige Maßnahmen des öffentlichen Gesundheitsdienstes,

\section{Abstract}

Background During a language course in Ireland 422 schoolchildren aged 15-17 years and 44 accompanying persons from the autonomous Province of Bolzano - South Tyrol in Italy were repeatedly exposed to a Mycobacterium tuberculosis complex culture-positive, but smearnegative Irish teacher for more than 10 lessons.

Methods Immediate action included contact tracing of exposed persons, administration of a standardised questionnaire, intradermal PPD (5IU) testing and thorax X-ray of persons with an induration of $\geq 5 \mathrm{~mm}$ in the PPD test. The questionnaire included information on demographic factors, symptoms, vaccination status, underlying diseases, regular medical treatment and past infection in the last three months. A control group of children with similar age and BCG vaccination status was enrolled in the same schools to estimate the effect of exposure and the influence of previous BCG vaccination and ambient non-tuberculous mycobacteria.

Results Exposure in Ireland was not associated with an elevated prevalence of positivity in the PPD test in the exposed group, but having intrafamiliar contact with tuberculosis cases (OR=3.3; 95\% confidence interval: $1.35-8.07$ ) or being from school $\mathrm{A}(\mathrm{OR}=2.3$; $95 \%$ confidence interval: 1.19-4.32) was associated with an induration $\geq 5 \mathrm{~mm}$ in the multivariate analysis.

Conclusions Contact tracing is an important public health action after exposure to an infectious tuberculosis case, nevertheless, in this case it may have resulted in overtreatment of exposed persons. BCG vaccination may have a long-term effect on PPD skin testing. 
auch wenn in diesem Fall mehr Exponierte behandelt wurden als notwendig gewesen wäre. Eine zurückliegende BCG-Impfung könnte einen Langzeiteffekt auf die intradermale Hauttestung haben.

\section{Einleitung}

Bei der Diagnose einer behandlungsbedürftigen Lungentuberkulose (Mycobacterium-tuberculosis-Komplex MT) sind die aktive Fallfindung im Umfeld des Kranken, zusammen mit der Behandlung desselben, Maßnahmen höchster Priorität in der Bekämpfungsstrategie gegen die Ausbreitung der Erkrankung [1-5]. Diese Umfeldkontrollen sollen nach der Entdeckung eines Patienten mit aktiver (behandlungsbedürftiger) Lungentuberkulose möglichst rasch durchgeführt werden, um die Neuinfektionen bzw. Neuerkrankungen frühzeitig erfassen, behandeln und somit die Übertragungskette unterbrechen zu können.

Insbesondere wird der Diagnose der LTBI (Latent Tuberculosis Infection) und deren Therapie von der WHO, Center of Disease Control (CDC), Advisory Council for the Elimination of Tuberculosis (ACET) und American Thoracic Society [6-8] eine große epidemiologische Bedeutung für die Tuberkuloseelimination in den Industrieländern zugeschrieben, denn durch die Behandlung der LTBI kann die Progression der Infektion zur aktiven tuberkulösen Erkrankung unterbunden werden. Dies gilt besonders bei Risikogruppen, denn diese weisen aus mehreren Gründen eine erhöhte Wahrscheinlichkeit auf, eine LTBI bzw. eine manifeste Erkrankung zu entwickeln.

Internationale wissenschaftliche Gesellschaften und das italienische Gesundheitsministerium haben Richtlinien für die korrekte Vorgehensweise bei Umfelduntersuchungen erlassen [2,9-10]. Die darin empfohlenen Standardmethoden zur Erfassung der LTBI bzw. der manifesten Erkrankung sind der Tuberkulintest nach Mendel-Mantoux mit 5IE PPD und die Thoraxröntgenuntersuchung. Damit die optimale Sensitivität in der Aussagekraft des Tuberkulintests für Personen, die einen engen Kontakt mit einem aktiven Lungentuberkulosefall aufweisen, erreicht werden kann, wird als Testkriterium (Cut-off point) ein Indurationsdurchmesser von $5 \mathrm{~mm}$ verwendet, d.h. Hautindurationen $\geq 5 \mathrm{~mm}$ werden als positiv angesehen [9-11]. Die Wiederholung der Screening-Untersuchungen wird nach einem Zeitintervall von 2 Monaten empfohlen. Bei dieser Signifikanzschwelle kann die Rolle der Umweltmykobakterien (nicht-tuberkulöse Mykobakterien - NTM), die ubiquitär, jedoch geografisch unterschiedlich, in der Umgebung des Menschen verbreitet sind, relevant störend sein. Diese NTM können für Kreuzreaktionen bzw. für eine Verminderung der Spezifizität und folglich des positiven prädiktiven Wertes verantwortlich sein und beeinflussen daher die Beurteilung der Tuberkulintestergebnisse im Hinblick auf die korrekte Dichotomisierung in MT-Infizierte und Nichtinfizierte [12-13]. Diese Störwirkung ändert sich stark nach geografischer Lage und kann somit auch zu unterschiedlichen Interpretationsschwierigkeiten der Tuberkulinreaktionen führen [14-15]. Aus diesem Grund bzw. um den realen MT-Expositionsgrad einer Gruppe besser definieren zu können, ist es hilfreich, die in einer Risikogruppe beobachtete Infektionsprävalenz mit der in der allgemeinen Bevölkerung erwarteten zu vergleichen [16]. Hinzu kann auch die Rolle einer eventuellen Sensibilisierung durch den Bazillus Calmette-Guérin (BCG) kommen [17-21]. Aus dem Vergleich dieser 2 Größen kann die störende
Wirkung von NTM und BCG-Impfung bei der Interpretation der Resultate minimiert werden.

In diesem Zusammenhang sei noch erwähnt, dass die Induration bei vorausgegangener, einmaliger BCG-Impfung gewöhnlich eine Größe von $10 \mathrm{~mm}$ nicht überschreitet. Sie erlischt meistens nach 10 Jahren [9,22-24], so dass eine vorausgegangene BCGImpfung eine untergeordnete Rolle im diagnostisch-therapeutischen Umgang mit Kontaktfällen spielen sollte [5].

Vierhundertsechsundsechzig in der autonomen Provinz Bozen Südtirol ansässige Schüler und Begleitpersonen hatten während eines einwöchigen Sprachaufenthaltes in Irland wiederholt Kontakt mit einem Lehrer einer irischen Sprachschule. Die Expositionsdauer betrug insgesamt mindestens 10 Unterrichtsstunden. Der Nachweis von Mycobacterium-tuberculosis-Komplex gelang beim Erkrankten zwar in der Sputumkultur, der Ausstrich aber war negativ. Die meisten Schüler wurden bei der Geburt BCG geimpft.

Ziel dieser Screeningstudie ist es, mögliche LTBI und Erkrankungsfälle in dieser Risikogruppe zu erfassen und zu therapieren, wobei die Störwirkung der NTM und der vorausgegangenen BCG-Impfung bei der Identifizierung der LTBI zu evaluieren und berücksichtigen ist.

\section{Material und Methoden}

Im September 2004 wurde die epidemiologische Beobachtungsstelle der autonomen Provinz Bozen - Südtirol von der Direktion der Abteilung für Epidemiologie und Biostatistik des italienischen Oberen Gesundheitsinstitutes (Istituto Superiore di Sanità) in Rom informiert, dass die irischen Behörden 466 in Südtirol ansässige Personen (Schüler und Begleitpersonen) ermittelt hatten, die in der Zeit vom 7. März 2004 bis 16. Mai 2004 anlässlich eines einwöchigen Sprachaufenthaltes Kontakt mit einem tuberkulosekranken Lehrer hatten. Die Expositionsdauer betrug insgesamt mindestens 10 Unterrichtsstunden. Den Südtiroler Behörden gelang es, 450 (97\%) dieser Kontaktpersonen (422 Schüler und 44 Begleitpersonen (40 Lehrer und 4 Eltern)) zu kontaktieren. Bei 445 Personen wurde ein Tuberkulintest durchgeführt. Von den 445 durchgeführten Tuberkulintests, waren 441 Personen (99\%), 402 Schüler und 39 Begleitperson,

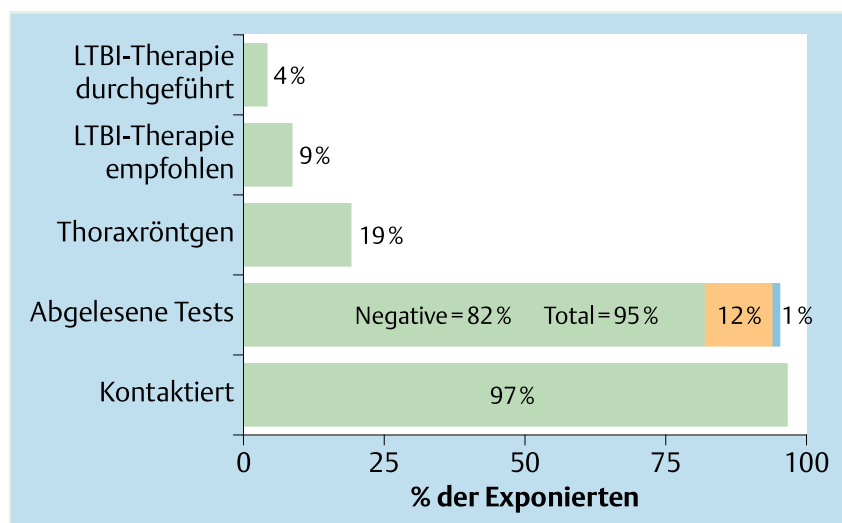

Abb. 1 „Epidemic Response“: Anteil der kontaktierten Personen, der durchgeführten und abgelesenen Tuberkulintests, der durchgeführten Thoraxröntgenuntersuchungen und der empfohlenen und durchgeführten LTBI-Therapien in exponierten Südtiroler Schülern und Begleitpersonen $(n=466)$. 
zur Ablesung erschienen. Von drei Schülern wurden die Tuberkulintestergebnisse auswärts erhoben und schriftlich mitgeteilt, daher wurden 444 Personen in die Analyse einbezogen (Abb. 1). Von den 16 Personen, die nicht daran teilnahmen, war ein Viertel Lehrpersonal $(n=4)$, drei der Schüler waren zurzeit der Untersuchung im Ausland (19\%) und einmal wurde die Teilnahme grundlos und ohne jede rechtliche Konsequenz verweigert; die meisten der restlichen Personen, die nicht an der Untersuchung teilnahmen, hatten die Schule gewechselt. Alle Erziehungsberechtigte bzw. Schüler wurden schriftlich über die Exposition und die notwendigen Schritte (Tuberkulintest und eventuelle Thoraxröntgenuntersuchung) benachrichtigt und aufgefordert, uns die Ergebnisse des Tuberkulintests sowie einer evtl. durchgeführten Thoraxröntgenuntersuchung mitzuteilen. In der Analyse wurden auch die auswärtig erhobenen Ergebnisse berücksichtigt.

Die Schüler und Begleitpersonen kamen von vier verschiedenen Schulen (A-D), die alle Oberschulen (Abschluss mit Abitur) verschiedener Fachrichtungen sind. Die Bezeichnungen A-D beziehen sich auf den Ort der Schulen, wobei Klassen der Schule A in der 12. Woche, der Schule B in der 13. und 15. Woche, der Schule $C$ in der 14. Woche und der Schule D in der 18., 20. und 21. Woche des Jahres 2004 in Irland waren.

Das mittlere Alter der Schüler war 17,3 Jahre (Tab. 3), das der Begleitpersonen 35,9 Jahre (18 - 50 Jahre). Unter den Schülern waren 50,4\% Mädchen, bei den Begleitpersonen 61,5\% ( $n=24)$. Die BCG-Durchimpfungsrate war $88,4 \%$ bei den Schülern $(n=358)$ und $42,1 \%(n=8)$ bei den Begleitpersonen.

Die Gruppen hatten während eines einwöchigen Sprachaufenthaltes in Irland mit einem irischen, an einer Lungentuberkulose erkrankten Lehrer wiederholt Kontakt in normalen Klassenzimmern, deren Fenster sich zum Lüften öffnen lassen. Die Expositionsdauer betrug insgesamt mindestens 10 Unterrichtsstunden. Der Nachweis von Mycobacterium-tuberculosis-Komplex gelang beim Erkrankten zwar in der Sputumkultur, der Ausstrich aber war negativ.

Der tuberkuloseerkrankte Lehrer konnte durch 2 Neuinfektionen und eine Erkrankung unter irischen Schülern und einen weiteren irischen Fall, der mit diesem Lehrer zusammengewohnt hatte, von den irischen Behörden identifiziert werden. Dies jedoch erst relativ spät, da er zum Zeitpunkt des Auftretens der Fälle unter irischen Schülern die Schule gewechselt hatte.

Kurz nach der Fallmeldung an die Südtiroler Gesundheitsbehörde wurde der Dienst, der für die Tuberkulose-Kontrolle zuständig ist, informiert und ein Plan für das weitere Vorgehen beschlossen. Die Öffentlichkeit wurde mittels einer Pressekonferenz informiert und eine Telefon-Hotline eingerichtet.

Es wurde eine Umfeldkontrolle (Contact tracing) geplant, bei der von allen Exponierten, nach Einholen einer Einverständniserklärung der Beteiligten (oder deren Eltern bei Minderjährigen), eine Anamnese mit einem standardisierter Fragebogen mittels eines persönlichen Interviews in den Schulen erhoben und ein Tuberkulintest nach Mantoux mit 5IE PPD durchgeführt wurde, der nach 72 Stunden von geschultem Personal abgelesen wurde. Im Fragebogen wurden von den Exponierten Informationen über demografische Faktoren (Alter, Geschlecht, Wohnort), Symptome (Fieber, Husten, Nachtschweiß, Gewichtsverlust), Impfstatus, Grunderkrankungen (Diabetes mellitus, Lymphom, Leukämie) regelmäßige Arzneimitteleinnahme (Corticoid-Therapie), während der letzten 3 Monate durchgemachte Infektionskrankheiten (Masern), eine mögliche familiäre Exposition und eine vorausgegangene BCG-Impfung erhoben, die anschließend in den Impfregistern der zuständigen Dienste verifiziert wurden. Des Weiteren wurden alle Personen, die im Hauttest einen Indurationsdurchmesser $\geq 5 \mathrm{~mm}$ zeigten, zu einer Thoraxröntgenaufnahme und pneumologischen Untersuchung eingeladen.

Allen Personen, bei denen die Indikation einer LTBI-Behandlung vorlag [9], wurde Isoniazid (INH) für 6 Monate empfohlen [10]. Angesichts der relativ lange zurückliegenden Exposition (Mittelwert 24,5 Wochen) wurde auf die Wiederholung dieser Vorsorgeuntersuchungen nach 2 Monaten verzichtet.

Aufgrund der hohen Durchimpfungsrate der exponierten Schüler bei der Geburt und um den vorhin erwähnten Vergleich zwischen erwarteter und beobachteter Infektionsprävalenz zu ermöglichen, wurde die exponierte Gruppe auch mit dem Testkriterium von 15 mm studiert bzw. eine Kontrollgruppe von Schülern untersucht, um auch die Spezifizität und den positiven prädiktiven Wert des Tuberkulintests zu erhöhen und somit die Störwirkung der Umweltmykobakterien und der BCG-Impfung besser abwägen zu können (der Großteil der Schüler hatte im Kleinkindesalter eine BCG-Impfung erhalten). Hierzu wurden 188 Schüler derselben Altersklasse ohne bekannte Tuberkuloseexposition und mit ähnlicher BCG-Durchimpfungsrate in Parallelklassen derselben Schulen nach Einholen einer Einverständniserklärung untersucht. Ein solches Vorgehen ist in Italien nach Einholen einer Einverständniserklärung möglich. Ein Tuberkulintest wurde vom selben Gesundheitspersonal durchgeführt. Die Kontrollpersonen waren ähnlich bezüglich des Geschlechts, jedoch geringfügig jünger als die exponierten Schüler (Tab. 3). Bei dieser Gruppe wurde eine Thoraxröntgenaufnahme, pneumologische Untersuchung und eventuelle INH-Prophylaxe erst bei einem Indurationsdurchmesser $\geq 15 \mathrm{~mm}$ empfohlen.

Alle Informationen wurden in eine elektronische Datenbasis eingegeben (epiinfo version 3.2, WHO, CDC, Atlanta) und analysiert. Für den Vergleich der Prävalenz zwischen der Gruppe der Exponierten mit der Gruppe der Nicht-Exponierten wurde ein ChiQuadrat-Test verwendet. Für die multivariate Analyse der exponierten Gruppe wurde eine unkonditionale logistische Regression verwendet; als Outcome wurde eine Hautinduration $\geq 5 \mathrm{~mm}$ als positiv angesehen; im multivariaten Modell wurden alle Faktoren berücksichtigt, die in der univariaten Analyse einen p-Wert unter 0,2 hatten. Im endgültigen multivariaten Modell waren nur Variablen enthalten, die eine statistisch signifikante Assoziation zwischen Exposition und Outcome aufwiesen.

\section{Ergebnisse}

\section{Tuberkulintestergebnisse}

Insgesamt wurden 444 Personen in der Analyse berücksichtigt, davon waren $91,2 \%$ Schüler $(n=405)$ und 8,8\% Begleitpersonen $(\mathrm{n}=39)$.

Der mittlere Durchmesser bei Schülern war 1,8 mm (Schwankungsbreite $0-20 \mathrm{~mm}$ ) und bei Begleitpersonen 2,0 mm (Schwankungsbreite 0-24 mm). Der Unterschied war aber nicht statistisch signifikant $(\mathrm{p}=0,236)$.

\section{Fünf-Millimeter-Testkriterium bei den exponierten Schülern und Begleitpersonen}

Wie vorhin erwähnt, empfehlen die internationalen Richtlinien für Kontaktpersonen ein Testkriterium von $5 \mathrm{~mm}$, d. h. ein Indurationsdurchmesser $\geq 5 \mathrm{~mm}$, damit das Tuberkulintestergebnis als positiv angesehen werden kann. 


\begin{tabular}{|c|c|c|c|}
\hline Faktor & OR & $95 \% \mathrm{Cl}$ & p-Wert \\
\hline BCG geimpft zu sein & 1,83 & $0,07-4,79$ & 0,22 \\
\hline BCG ungeimpft zu sein & Ref. & & \\
\hline Familienmitglied mit Tb & 3,00 & $1,24-7,22$ & 0,01 \\
\hline Kein Kontakt mit Tuberkuloseerkrankten in der Familie & Ref. & & \\
\hline Zumindest an einem Symptom seit Exposition zu leiden & 0,70 & $0,33-1,42$ & 0,34 \\
\hline Symptomlos zu sein & Ref. & & \\
\hline (Orale oder) systemische Kortikoid-Therapie & 1,55 & $0,17-14,06$ & 0,70 \\
\hline Keine Kortikoid-Therapie & Ref. & & \\
\hline Schule A (Brixen) & 2,11 & $1,12-4,00$ & 0,02 \\
\hline Andere Schule & Ref. & & \\
\hline Schule B (Bozen) & 0,69 & $0,36-1,29$ & 0,24 \\
\hline Andere Schule & Ref. & & \\
\hline Schule C (Schlanders) & 1,02 & $0,55-1,89$ & 0,94 \\
\hline Andere Schule & Ref. & & \\
\hline Schule D1 (Meran 1) & 0,60 & $0,25-1,45$ & 0,26 \\
\hline Andere Schule & Ref. & & \\
\hline Schule D2 (Meran 2) & 1,05 & $0,51-2,19$ & 0,90 \\
\hline Andere Schule & Ref. & & \\
\hline Schüler zu sein & 1,11 & $0,42-2,97$ & 0,83 \\
\hline
\end{tabular}

Tab. 1 Faktoren, die in der univariaten Analyse mit einem Indurationsdurchmesser $\geq 5 \mathrm{~mm}$ assoziiert waren (Schüler; $\mathrm{n}=405$ )

\begin{tabular}{llll} 
Faktor & OR & $\mathbf{9 5 \%} \mathbf{C l}$ & p-Wert \\
Familienmitglied mit Tb & 3,31 & $1,35-8,07$ & 0,01 \\
\hline Schule A (Brixen) & 2,27 & $1,19-4,32$ & 0,01
\end{tabular}

Bei diesem als sehr sensitiv zu bezeichnenden Kriterium war die Prävalenz der tuberkulinpositiven Schüler $(14,0 \% ; n=57)$ ähnlich hoch wie diejenige der Begleitpersonen $(12,8 \% ; n=5)$.

In der univariaten Analyse waren 2 Faktoren mit einem Indurationsdurchmesser $\geq 5 \mathrm{~mm}$ assoziiert (Tab. 1). Diese Assoziation änderte sich nur unwesentlich, als auch die anderen Faktoren in das multivariate Modell eingefügt wurden. Sowohl die Angabe, dass ein Familienmitglied an einer Tuberkulose $(\mathrm{Tb})$ in der Vergangenheit erkrankt war, als auch die Tatsache die Oberschule A besucht zu haben, waren mit einer Odds Ratio von annähernd 3,3 bzw. 2,3 im multivariaten Modell mit einem Indurationsdurchmesser $\geq 5 \mathrm{~mm}$ assoziiert (Tab. 2).

\section{Fünfzehn-Millimeter-Testkriterium bei den exponierten} Schülern und Begleitpersonen

Von 405 Schülern und 39 Begleitpersonen, bei denen der Test durchgeführt und abgelesen werden konnte, sind $0,7 \%$ der Schüler $(n=3)$ und 8,2\% der Begleitperson ( $n=3)$ mit dem Fünfzehn-

$\begin{array}{ll}\text { Kontrollgruppe } & \text { p-Wert } \\ 48,1 & (0,91) \mathrm{NS} \\ 16,7 & <0,001 \\ (17 ; 15-25) & \\ 173 / 187 & (0,13) \mathrm{NS} \\ 92,5 \% & \\ 1,68 \mathrm{~mm} & (0,66) \mathrm{NS} \\ (0 ; 0-18 \mathrm{~mm}) & \\ & \\ 30 / 188 & (0,55) \mathrm{NS} \\ 16,0 \% & \\ 3 / 188 & \\ 1,6 \% & (0,33) \mathrm{NS}\end{array}$

Tab. 3 Vergleich von demografischen Parametern, BCGDurchimpfungsrate und Hautreaktion des Mantouxtests zwischen exponierten Schülern und nicht-exponierten Schülern.

BCG-Durchimpfungsrate und Tuberkulinindizes mittels des Fünf-Millimeter- und Fünfzehn-Millimeter-Testkriteriums im Vergleich zwischen exponierten und nicht-exponierten Schülern
Millimeter-Testkriterium als positiv anzusehen: einer der 3 Schüler (OR = 8,52; $95 \%$ CI: 0,14-169,69; $p=0,03)$ und zwei der drei Begleitperson, die einen positiven Tuberkulintest mit dem Fünfzehn-Millimeter-Testkriterium zeigten, gaben an, einen Familienangehörigen mit Tuberkulose gehabt zu haben.

Die Assoziation zwischen Positivität und der Tatsache, dass ein Familienmitglied früher an Tuberkulose erkrankt war (der Zeitraum konnte meist nicht angegeben werden), nahm umgekehrt proportional mit der Sensitivität zu (3faches Risiko mit dem Fünf-Millimeter- und 8fach mit dem Fünfzehn-Millimeter-Testkriterium).

Der zweite Faktor, der eine Positivität im Tuberkulintest mit dem Fünf-Millimeter-Testkriterium, sowohl in der univariaten als auch multivariaten Analyse beeinflusste, war die Schule (Tab. 1 und 2). Der Durchschnitt der Positivität mit dem Fünf-Millimeter-Testkriterium lag bei $14 \%$, mit Variationsbreite zwischen $4 \%$ (Schule C) und 33\% (Schule A). 


\section{BCG-Impfung}

Insgesamt wurden 424 exponierte Personen (Schüler $n=405$; Begleitperson $\mathrm{n}=19$ ) zur Analyse der BCG-Durchimpfungsrate herangezogen. Diese lag bei $88,4 \%$ bei Schülern $(n=358)$ und bei $42,1 \%$ bei Begleitpersonen $(n=8)$. Eine vorhergehende BCGImpfung war nicht mit einer Positivität des Tuberkulintests mit dem Fünf-Millimeter-Testkriterium in der univariaten Analyse assoziiert und wurde daher nicht in das multivariate Modell mit einbezogen (OR der BCG-Impfung in Tab. 1).

\section{Thoraxröntgenuntersuchung}

Insgesamt wurden 89 Personen einer Thoraxröntgenuntersuchung unterzogen (Abb. 1), die alle keine tuberkulösen, pleuroparenchymalen bzw. mediastinalen Veränderungen aufwiesen. Eine Person, die eine Induration $\geq 5 \mathrm{~mm}$ hatte, hat keine Thoraxröntgenuntersuchung durchgeführt. 28 Personen mit einem negativen Tuberkulintestergebnis $(<5 \mathrm{~mm})$ wurden hingegen einer Thoraxröntgenuntersuchung unterzogen, davon 7 Personen, weil sie bei Exposition unter 16 Jahre alt waren, einer wegen einer immunsuppressiven Therapie, 3 wegen Nachtschweiß, einer wegen Gewichtsverlust und 16 wegen eines bestehenden Hustens.

\section{LTBI-Behandlung}

Es wurde 41 Personen (39 Schüler (9,7\% der abgelesenen) und 2 Begleitpersonen (5,1\% der abgelesenen)) eine Behandlung der LTBI empfohlen. 20 Schüler, das sind 51,3\% der Schüler, denen eine Therapie der LTBI vorgeschlagen wurde, und 35,1\% der Schüler, die eine Induration $\geq 5 \mathrm{~mm}$ hatten, waren mit der Therapie einverstanden und haben die Therapie begonnen (Abb. $\bullet 1)$.

\section{Ergebnisse der nicht-exponierten Gruppe}

Unter Anwendung des Fünf-Millimeter- und Fünfzehn-Millimeter-Testkriteriums betrug die Prävalenz der tuberkulinpositiven Schüler in der nicht-exponierten Gruppe jeweils 16,0\% ( $n=30)$, und 1,6\% $(n=3)$. Darüber hinaus ergab der Vergleich dieser Prävalenzen in der exponierten und nicht-exponierten Gruppe keine statistisch signifikanten Unterschiede $(p=0,55)$ bei den verwendeten Testkriterien (Abb. @ 2).

Zum Abschluss wurde eine Schätzung der Langzeitwirkung der BCG-Impfung durchgeführt, indem alle Studienteilnehmer (Exponierte und Nicht-Exponierte), die keinen Tuberkulosefall in der Familie angegeben haben, verglichen wurden. Dies ergab, dass geimpfte Schüler doppelt so häufig eine Induration $\geq 5 \mathrm{~mm}$ zeigten wie nicht-geimpfte Schüler (Abb.๑3), dieser Unterschied ist jedoch nicht als statistisch signifikant zu betrachten $(\mathrm{p}=0,11)$.

\section{Diskussion}

Ziel dieser Screening-Studie war es, nach Kontakt mit einem tuberkulösen Indexpatienten mögliche LTBI und Folgeerkrankungen in einer Risikogruppe von Jugendlichen und Erwachsenen zu erfassen und zu therapieren, wobei die Störwirkung der vorausgegangenen BCG-Impfung und der NTM bei der Identifizierung der LTBI-Fälle evaluiert und berücksichtigt werden musste. Der Unterschied zwischen den Tuberkulinindizes der exponierten und nicht-exponierten Schüler mit dem Fünf-Millimeter-Kriterium (jeweils 14\% und 16\%) und mit dem FünfzehnMillimeter-Testkriterium (jeweils 0,7\% und 1,6\%) ist zum großen Teil auf die störende Wirkung der NTM und BCG-Sensibilisie-

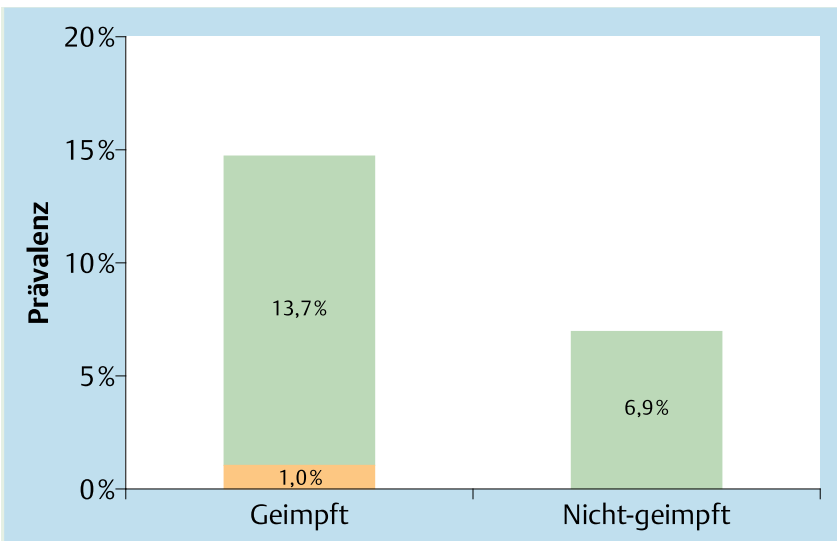

Abb. 2 Vergleich der Indurationsdurchmesser $\geq 5 \mathrm{~mm}$ und $\geq 15 \mathrm{~mm}$ von exponierten Südtiroler Schülern $(n=444)$ und einer Kontrollgruppe von Schülern $(n=188)$.

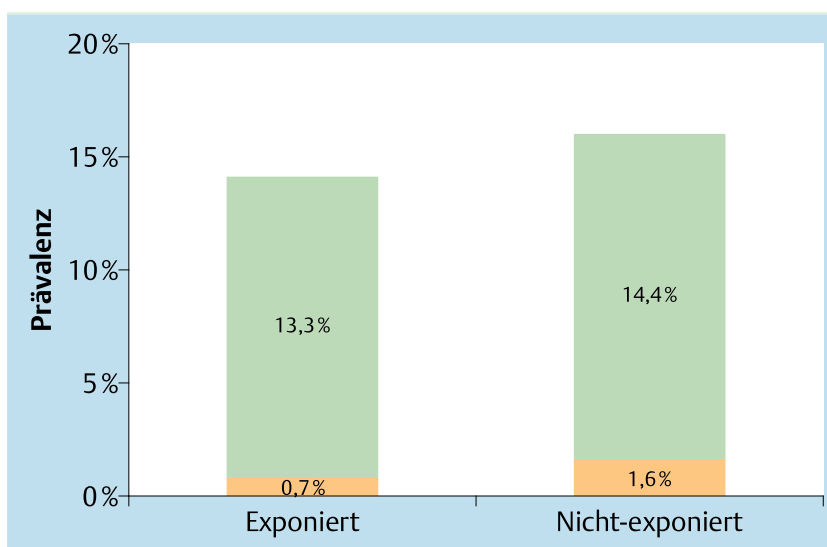

Abb. 3 Vergleich der Indurationsdurchmesser $\geq 5 \mathrm{~mm}$ und $\geq 15 \mathrm{~mm}$ von geimpften ( $n=498)$ und nicht-geimpften Südtiroler Schülern $(n=58)$, die nach ihren Angaben keinen Tb-Kranken in der Familie hatten. NS: nicht signifikant

** einer der drei exponierten Schüler mit Reaktionsdurchmesser $\geq 15 \mathrm{~mm}$ hatte einen Kontakt mit einem Tb-Kranken innerhalb der Familie, die anderen beide waren geimpft. Die Thoraxröntgenuntersuchung fiel jeweils negativ aus. Empfohlen wurden eine LTBI-Therapie sowie eine Röntgenkontrolle.

rung zurückzuführen. Über dieses Phänomen wird auch in der Literatur des Öfteren berichtet [25-29].

Der Vergleich obgenannter Tuberkulinindizes und die Tatsachen, dass in der exponierten Gruppe von Schülern gegenüber der nicht-exponierten Gruppe häufiger angegeben wurde, dass bei einem Familienangehörigen eine Tuberkulose diagnostiziert wurde $(9,2 \%$ versus $6,2 \% ; p=0,34)$, jedoch nicht häufiger eine Positivität (weder mit dem Fünf- noch mit dem Fünfzehn-Millimeter-Testkriterium) beobachtet wurde, unterstreicht die Vermutung, dass die Exposition der Schüler in Irland wahrscheinlich nicht mit einem erhöhten Risiko für Tuberkulose assoziiert war. Keine genauere Aussage über den wahren Expositionsgrad lässt sich hingegen für die Gruppe der Begleitpersonen machen, denn in diesem Fall wurde keine nicht-exponierte vergleichbare Kontrollgruppe herangezogen.

Aus diesem Grund liegt die Vermutung nahe, dass die a priori getroffenen diagnostischen (Thoraxröntgenuntersuchung) und therapeutischen Vorsorgemaßnahmen (LTBI-Behandlungen), 
welche zum großen Teil auf Informationen der irischen Behörde fußen, a posteriori wahrscheinlich nicht indiziert waren. Diese Behauptung gilt besonders für die Schüler. Mitverantwortlich für diesen Umstand ist die Tatsache, dass bei der Fallmeldung lediglich Informationen über die Infektiosität des TuberkuloseFalles vorlagen, jedoch keine genauere Daten über diejenigen Variablen - d.h. Kontaktbedingungen, Volumen und Belüftung der Schulzimmer usw. - zur Verfügung standen, welche die tatsächlichen Kontaktmerkmale zwischen dem tuberkuloseerkrankten Lehrer und den Exponierten und somit den Expositionsgrad näher definieren. Diese Kontaktmerkmale sind stets bei der Programmierung von Umfelduntersuchungen und für die Erstellung der Liste der Kontaktpersonen zu eruieren und im Hinblick auf das korrekte Prozedere im Rahmen einer Umfeldkontrolle zu berücksichtigen [8-9].

Die Tatsache, dass in der Schule A eine erhöhte Prävalenz mit einer Induration $\geq 5 \mathrm{~mm}$ beobachtet wurde, könnte dadurch bedingt sein, dass andersartige Kontaktmerkmale in der Schule A vorherrschten (es wurde von den Schülern dieser Schule angegeben, dass sie auch während der Freizeit mehrere Ausflüge mit irischen Lehrpersonen unternommen haben oder aber auch, dass in dieser Schule in früheren Jahren eine verstärkte Exposition mit einem Tuberkulosefall möglich war, da sowohl eine Mutter als auch ein Vater von jeweils einem Schüler der Schule A an Tuberkulose erkrankt waren).

Mit dem Fünf-Millimeter-Testkriterium war die Positivität nicht signifikant unterschiedlich zwischen Begleitpersonen, exponierten und nicht-exponierten Schülern. Dies ist wahrscheinlich dadurch bedingt, dass die NTM-Sensibilisierung der Südtiroler Gesamtbevölkerung einen gewichtigen Störfaktor darstellt und dass deutlich mehr Schüler BCG geimpft waren als Begleitpersonen. Die störende Rolle der BCG-Impfung wird auch dadurch unterstrichen, dass geimpfte Schüler doppelt so häufig eine Induration $\geq 5 \mathrm{~mm}$ aufwiesen wie nicht-geimpfte Schüler, auch wenn diese Beobachtung aufgrund der geringen Anzahl von nichtgeimpften Schülern nicht statistisch signifikant war. Aufgrund der begrenzten Anzahl der Schüler in den Parallelklassen und der limitierten menschlichen Ressourcen, konnte die Kontrollgruppe nicht beliebig vergrößert werden, um dieses Phänomen genauer zu untersuchen. Obwohl in der internationalen Literatur angegeben wird, dass eine BCG-Impfung nach mehr als 10 Jahren bezüglich einer positiven Hautreaktion vernachlässigbar sei $[11,15,18,28]$, kann nicht ausgeschlossen werden, dass eine zum Zeitpunkt der Geburt verabreichte BCG-Impfung auch nach mehr als 15 Jahren einen deutlichen Einfluss auf ein Tuberkulintestergebnis hat.

Bei abnehmender Erkrankungsprävalenz in der Allgemeinbevölkerung konzentrieren sich die Kontrollmaßnahmen gegen die Tuberkulose zunehmend auf die Erfassung der Erkrankungsfälle und der LTBI in Risikogruppen. Der Tuberkulintest stellt in diesem Rahmen ein wichtiges diagnostisches Mittel dar, welches jedoch aufgrund der mangelnden Spezifizität schnell an seine Grenzen stößt. Die Einführung neuer Untersuchungsmethoden zur Diagnose der LTBI, welche eine größere Spezifizität, d.h. keine Kreuzreaktionen mit den Antigenen von NMT und BCG, aufweisen und gezielt in Zweifelsfällen eingesetzt werden können, vermag den Einsatz von Vorsorgemaßnahmen für die Diagnose und Behandlung der LTBI effizienter und wirksamer zu gestalten [30-33], standen jedoch nicht zur Verfügung.

\section{Literatur}

1 Jakubowiak Wet al: TB-Manual: National Tuberculosis Programme Guidelines. WHO EURO 2001

2 American Thoracic Society: Diagnostic standards and Classification of Tuberculosis in Adults and Children. Am J Respir Crit Care Med 2000; 161: $376-1395$

3 Broeckmans JF, Migliori GB, Rieder HLet al: European Framework for tuberculosis control and elimination in countries with a low incidence. Eur Respir J 2002; 19: 765 - 775

4Centers of Disease Control and Prevention: Tuberculosis Elimination Revisited: Obstacles, Opportunities and a renewed Commitment. MMWR 1999; 48: 1-13

5 American Thoracic Society: Control of Tuberculosis in the United States. Am Rev Respir Dis 1992; 145: 1623 - 1633

6World Health Organisation: WHO Tuberculosis Programme: framework for effective tuberculosis control. Geneva: WHO 1994; WHO/ $\mathrm{TB} / 94,179$

7 Centers of Disease Control and Prevention: Recommendations of the ACET. Essential Components of a Tuberculosis Prevention and Control Program. MMWR 1995; 44: 1 - 34

8 American Thoracic Society: Control of Tuberculosis in the United States. Am Rev Respir Dis 1992; 145: 1623 - 1633

9 Centers of Disease Control and Prevention: Targeted tuberculin testing and treatment of latent tuberculosis infection. MMWR 2000; 49: 1 51

10 Ministero della Sanità: Decreto Legislativo 31 marzo 1998, n 112. Linee guida per il controllo della malattia tubercolare, su proposta del Ministero della sanità, ai sensi dell'art. 115, comma 1, lettera b.

11 American Thoracic Society: The tuberculin skin test. Am Rev Respir Dis 1981; $124: 346$ - 351

12 Snider DE: The tuberculin skin test. Am Rev Respir Dis 1982; 125 : $108-118$

13 Huebner RE, Schein MF, Bass JBJ: The tuberculin skin test. Clin Infect Dis 1993; 17: $968-975$

14 Rieder HL: Methodological issues in the estimation of the tuberculosis problem from tuberculin surveys. Tuberc Lung Dis 1995; 76: 114-121

15 Pretto P: Teoria e pratica nell'uso del test tubercolinico. Rass pat app resp 2003; 18: 34-45

16 Veen J: Tuberculosis control programmes in developed countries. Eur Respir Mon 1997; 4: 88-115

17 Dautzenberg B, Pariente R, Gicquel Bet al: A propos du BCG. Presse Méd 1997; 26: $273-276$

18 Cohn DL: The effect of BCG Vaccination on Tuberculin Skin Testing. Am J Respir Crit Care Med 2001; 164: 915-916

19 Chee CBE, Soh CH, Boudville ICet al: Interpretation of the tuberculin skin test in Mycobacterium bovis BCG-vaccinated Singaporean schoolchildren. Am J Respir Crit Care Med 2001; 164: 958 - 961

20 Singh SD, Sutton CJ, Woodcock AA: Repeat tuberculin testing in Mycobacterium tuberculosis BCG-vaccinated subjects in the UK: the booster effect varies with the time of reading. Am J Respir Crit Care Med 2001; 164: 962 - 964

21 Snider Jr DE: BCG and tuberculin skin tests. JAMA 1985; 253: 438 3439

22 Pretto P: L'indice tubercolinico nella popolazione della Provincia di Bolzano. Riv Pat Clin Tuberc e Pneumol 1989; 60: 93 - 104

23 Pretto P, Marchese Ragona A: La situazione epidemiologica tubercolare ed il monitoraggio degli esiti del trattamento antitubercolare in Alto Adige. Rass pat app resp 2001; 16: $343-350$

24 Anonymous: Red Book for PDA: Report of the Committee for Infectious Diseases. Academy of Pediatrics 2003

25 Rust P, Thomas J: A method for estimating the prevalence of tuberculous infection. Am J Epidemiol 1975; 101: 311 - 322

26 Menzies D: Interpretation of repeated tuberculin tests. Am J Respir Crit Care Med 1999; 159: 15-21

27 Huebner RE, Schein MF, Bass JBJ: The tuberculin skin test. Clin Infect Dis 1993; 17: $968-975$

28 Rieder HL: Theoretische und praktische Überlegungen bei Anwendung des Tuberkulintests. Pneumologie 1997; 51: 1025- 1032

29 Probst G, Apfel T, Schulz Vet al: Diagnostik, Therapie und Prognose atypischer Mykobakteriosen - Ergebnisse einer retrospektiven Studie. Pneumologie 1994; 48: 711 - 717

30 Desem N, Jones SL: Development of a human gamma interferon enzyme immunoassay and comparison with tuberculin skin testing for detection of Mycobacterium tuberculosis infection. Clin Diagn Lab Immunol 1998; 5: 531 - 536 
31 Mazurek GH, LoBue PA, Daley CLet al: Comparison of a whole-blood Interferon $\gamma$ assay with tuberculin skin testing for detecting latent Mycobacterium tuberculosis infection. JAMA 2001; 286 (14): 1740 - 1747

32 Streeton JA, Desem N, Jones SL: Sensitivity and specificity of a gamma interferon blood test for tuberculosis infection. Int J Tuberc Lung Dis 1998; 2 : $443-450$
33 Lalvani A, Pathan AA, McShane Het al: Rapid detection of Mycobacterium tuberculosis infection by enumeration of antigen-specific T-cells. Am J Respir Crit Care Med 2001; 163: 824-828 